Document downloaded from:

http://hdl.handle.net/10251/99755

This paper must be cited as:

Anza Hormigo, S.; Vicente Quiles, CP.; Gimeno Martinez, B.; Boria Esbert, VE.; Armendariz, J. (2007). Long-term multipactor discharge in multicarrier systems. Physics of Plasmas. 14(8):1-8. doi:10.1063/1.2768019

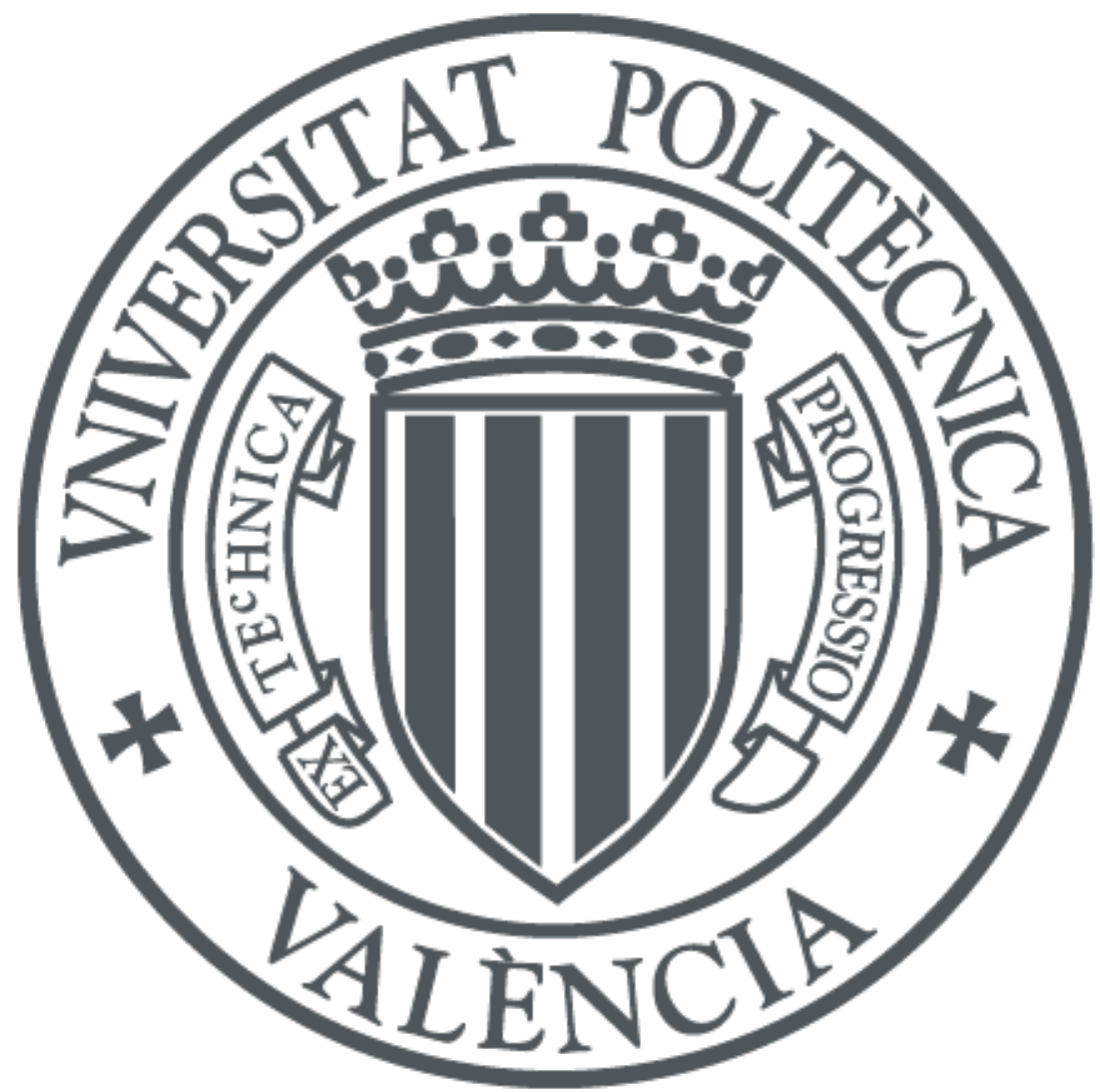

The final publication is available at https://doi.org/10.1063/1.2768019

Copyright American Institute of Physics

Additional Information 


\title{
Long-term multipactor discharge in multicarrier systems
}

\author{
S. Anza $a^{a)}$ \\ Aurora Software and Testing S.L., Parque Científico Universitat de València, \\ Polígono "La Coma" s/n, 46980 Paterna, Spain \\ C. Vicente \\ Departamento de Comunicaciones, Universidad Politécnica de Valencia Camino de Vera s/n, \\ 46022 Valencia, Spain \\ B. Gimeno \\ Departamento de Física Aplicada y Electromagnetismo - ICMUV, Universitat de València c/ Dr. Moliner, \\ 50, 46100 Valencia, Spain \\ V. E. Boria \\ Departamento de Comunicaciones, Universidad Politécnica de Valencia Camino de Vera s/n, \\ 46022 Valencia, Spain \\ J. Armendáriz \\ Aurora Software and Testing S.L., Parque Científico Universitat de València, \\ Polígono "La Coma” s/n, 46980 Paterna, Spain
}

\begin{abstract}
A new mechanism of long-term multipactor in multicarrier systems is studied employing both analytical and numerical methods. In particular, the investigation is focused on the impact that a realistic secondary emission yield at low energies produces on the development of long term multipactor. A novel analytical model for this interperiod charge accumulation is presented using the traditional multipactor theory for parallel plates, and approximating the multicarrier signal as a single-carrier signal modulated by a pulsed signal envelope. The analytical predictions are verified by numerical simulations for a typical rectangular waveguide. The analytical and numerical results demonstrate that the susceptibility of the system to develop a long-term multipactor discharge increases with higher values of low-energy secondary emission yield.
\end{abstract}

\section{INTRODUCTION}

Multipactor $^{1-3}$ is a nonlinear effect that may occur in high power microwave devices at very low pressures, such as those operating in particle accelerators and satellite subsystems. A multipactor discharge is an electronic avalanche, in synchronism with the rf field, caused by secondary emission multiplication on the device walls. Its effects range from signal degradation to the complete destruction of the component.

The design of multipactor-free components is a key issue for the space telecommunications industry. Specifically, new satellite payloads operate with an extremely high number of communication channels and a still increasing power level per carrier. This leads to extremely high peak power levels in the multicarrier path of the spacecraft, which results in an increasing risk of multipactor discharge. ${ }^{4,5}$

The study of multipactor in multicarrier operation is much more complex than in the single-carrier case. A typical multicarrier signal is composed of several modulated carriers with small frequency separation. Their mix produces a modulated rf signal with a time varying periodic envelope. Such signals add a dynamical behavior to the multipactor process, which is not well understood as yet. Currently, a

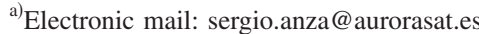

theory for multipactor in multicarrier operation is not well established.

Up to now, the standard adopted by the European Space Agency (ESA) for multicarrier multipactor design ${ }^{6}$ is based on the "20-gap-crossing rule," which states that multipactor takes place only if the multicarrier signal envelope exceeds the breakdown voltage for a time equal to or higher than the time that an electron takes to cross the gap 20 times $\left(T_{20}\right)$. This rule establishes a multipactor criterion for a single multicarrier peak. Nevertheless, the variation in time of the signal amplitude implies that, within one period of the multicarrier envelope, there are some intervals in which the amplitude of the field is above the multipactor breakdown threshold and others in which it is below. In the first case, the multipactor resonance and secondary emission yield (SEY) conditions are met, resulting in an exponential growth of the electron population. In the second case, the electrons step out of resonance and hit the walls with much lower energies and a SEY below 1, being therefore absorbed. The rate of absorption depends directly on the value of the SEY at such low energies. Therefore, if the total absorption is less than the total electron emission, there will be an overall growth or charge accumulation in one period. In such cases, since these multipactor activity and relaxation intervals, or "ON" and "OFF" intervals, are periodically repeated in time, the total electron population rises indefinitely until saturation after 
some periods, producing a multipactor discharge even though the 20-gap-crossing rule is accomplished.

Whereas traditional SEY models for multipactor characterization $^{7}$ decrease rapidly to zero at low energies, recent works on secondary emission effect support a nonzero value of the SEY at low energies (in the range of $\mathrm{eV}$ ) due to the presence of elastic electrons. ${ }^{8-11}$ In this work, a recently published SEY model ${ }^{12}$ is adopted, taking different values of the curve for low energies. On the other hand, the spread of the emission energy of the secondary emitted electrons is taken into account in both the analytical and the numerical analyses, since it results also in a spread in the electron impacting energy and, therefore, an increase in the range of valid phases at the end of the OFF interval. ${ }^{13}$ This presumably enhances the probability of the electron to participate in the multipactor process of the consecutive ON interval.

There are few works dealing with multipactor in multicarrier systems and accumulation. A possible justification for not existing experimental results reporting accumulation is that multipactor experiments with multicarrier signals are difficult and costly to set up due to the need for several high power amplifiers. In addition, the current multipactor detection methods do not allow one to determine the nature of the multipactor discharge. ${ }^{5,14,15}$ In Ref. 16, numerical results of electron population growth for multicarrier signals and infinite parallel plates are presented, but no accumulation is reported. On the other hand, a numerical study of multipactor with a two carrier signal is given by Semenov et al. in Ref. 4 where accumulation is present in some cases, although not treated in detail.

In this paper, a mechanism of long-term multipactor in multicarrier applications is addressed, and the impact of the SEY properties on multipactor accumulation is investigated for the first time. Firstly, a theoretical analysis of the electron population dynamics under the action of multicarrier signal fields is developed, where the multicarrier signal envelope is simplified and approximated to be an ON/OFF pulsed signal, which allows us to study the electron multiplication and absorption separately. The former is modeled by the classical parallel-plate two-surface single-carrier multipactor theory, whereas for the latter, and since there is no applied field, it is reasonable to assume that the electrons travel between the waveguide walls with constant velocity equal to the secondary electron emission one. This analytical model predicts the minimum average SEY needed to develop a charge accumulation process between consecutive periods of the envelope, resulting in a long-term multipactor discharge. Although the theory has been developed for pulsed signals, the analytical predictions are proven to be valid also for real multicarrier envelopes, provided that their envelope is reasonably well fitted by a pulsed signal. Finally, by means of an ad hoc multipactor simulator for a rectangular waveguide of infinite length and multicarrier input signal, the analytical predictions are verified for different values of the SEY at low energies.

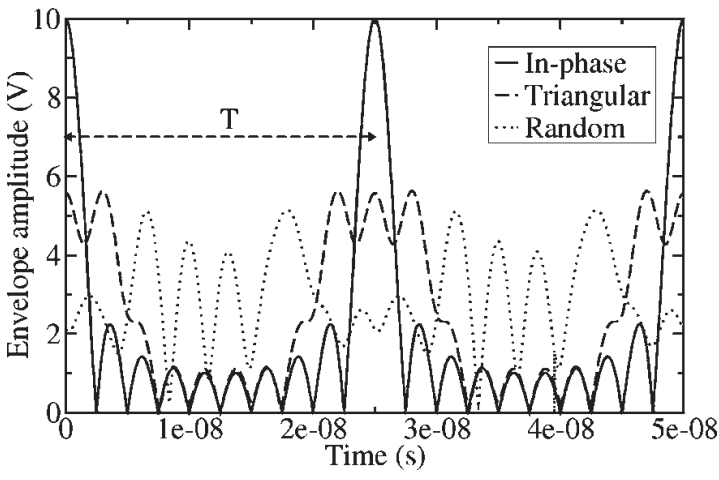

FIG. 1. Envelopes of a ten-carrier signal with a frequency separation of $\Delta f=40 \mathrm{MHz}$ and three different phase distributions with $V_{0}=1 \mathrm{~V}$. The period is $T=1 / \Delta f=25 \mathrm{~ns}$.

\section{MULTICARRIER MULTIPACTOR BASICS}

Multipactor takes place when the electromagnetic field and the geometry of the component are such that electrons follow a resonant trajectory, impacting consecutively from wall to wall of the device, with the adequate impact energy to ensure a SEY higher than 1. ${ }^{1}$ According to the classical single-carrier multipactor theory, ${ }^{2,3,17}$ in order to achieve resonance, the electron travel phases between plates must be an odd number of half-periods, i.e., $n \pi$, where $n=1,3,5,7, \ldots$ is the multipactor order. The multipactor order can be expressed analytically as a function of the applied voltage and the operation frequency times the device gap; i.e., $f \times d$. Analogously, the theory sets the value of the breakdown voltage $V_{B}$ (the minimum voltage to induce a multipactor discharge), as a function of the $f \times d$ and the SEY properties of the device material.

On the other hand, in typical communication applications, a multicarrier signal is composed by $N$ carriers, with same amplitude $V_{0}$, equally spaced in frequency $(\Delta f)$ and with relative phase $\varphi_{i}$, where $i$ stands for each individual carrier:

$$
v(t)=V_{0} \sum_{i=1}^{N} \sin \left\{2 \pi\left[f_{m}+\left(i-\frac{N+1}{2}\right) \Delta f\right] t+\varphi_{i}\right\},
$$

where $v(t)$ is the voltage of the compound signal and $f_{m}$ is the mean frequency of all carriers. Under these circumstances, the compound signal can be expressed as a single carrier with frequency $f_{m}$, modulated by an envelope, whose time evolution depends on the relative phases between carriers. ${ }^{18}$ The absolute value of the envelope is periodic with $T=1 / \Delta f$ period. Figure 1 shows an example of three different envelopes of an $N=10$ carrier signal of $V_{0}=1 \mathrm{~V}$ amplitude each and with $\Delta f=40 \mathrm{MHz}$, for three phase distributions. Notice that the in-phase case gives the maximum envelope peak, equal to $10 \mathrm{~V}$, and the narrower main lobe. On the contrary, the triangular distribution ${ }^{19}$ gives a lower but wider main lobe, whereas the arbitrary distribution can lead to any envelope ranging from the in-phase case to the situation where the envelope amplitude is almost equally distributed throughout the whole period (very similar to singlecarrier operation). 
In one period of the envelope, the signal amplitude may be either higher or lower than the single-carrier multipactor breakdown threshold $V_{B}$ (for $f_{m}$ ). Whereas in the first case there is electron multiplication, in the second one electrons are mainly absorbed. These two processes are repeated periodically with period $T$, where the rate of electron creation and absorption is governed mainly by the geometry of the device, the material SEY properties, and the amplitude of the envelope.

It is not straightforward to define a multipactor criterion under such conditions. In the classical single-carrier case, if the amplitude of the field is higher than $V_{B}$, the electron population grows indefinitely until saturation, certainly causing a multipactor discharge. However, in the multicarrier case, the electron population shows peaks and valleys, not being clear the correspondence between the peak height and the existence of the discharge.

The 20-gap-crossing rule ${ }^{6}$ establishes a multipactor criterion for multicarrier signals based in the peak height and duration of the multicarrier signal envelope. This rule has been obtained from numerical simulations and claims that multipactor takes place if the ON interval is longer than the 20-gap-crossing time $T_{20}$, which can be expressed as

$$
T_{20}=\frac{10 n}{f_{m}},
$$

$n$ being the multipactor order and $f_{m}$ the mean frequency of the multicarrier signal. Physically, $T_{20}$ corresponds to the time that an electron, under resonance conditions, takes to cross the gap 20 times. This criterion only considers the electron growth in one single event or envelope period, disregarding possible interperiod charge accumulation. In such cases the electron population curve may show an increasing exponential trend, thus producing a long-term multipactor discharge.

\section{SEY MODEL}

One of the most employed SEY models is the Vaughan's model. ${ }^{7}$ Vaughan proposed a parametric formula of the SEY which allows one to fit experimental data:

$$
\begin{aligned}
& \sigma(W, \theta)=\sigma_{\max }(\theta)\left(u e^{1-u}\right)^{k} \quad \text { for } u \leq 3.6, \\
& \sigma(W, \theta)=\sigma_{\max }(\theta) 1.125 / u^{0.35} \quad \text { for } u>3.6,
\end{aligned}
$$

where

$$
\begin{aligned}
& u=\frac{W-W_{0}}{W_{\max }(\theta)-W_{0}}, \\
& k=0.56 \text { for } u<1, \\
& k=0.25 \text { for } 1<u<3.6, \\
& \sigma_{\max }(\theta)=\sigma_{\max }\left(1+k_{E} \theta^{2} / 2 \pi\right), \\
& W_{\max }(\theta)=W_{\max }\left(1+k_{\theta} \theta^{2} / 2 \pi\right) .
\end{aligned}
$$

These equations give the value of the SEY $\sigma(W, \theta)$ for an electron impacting energy $W$ and incident angle $\theta$ with re-

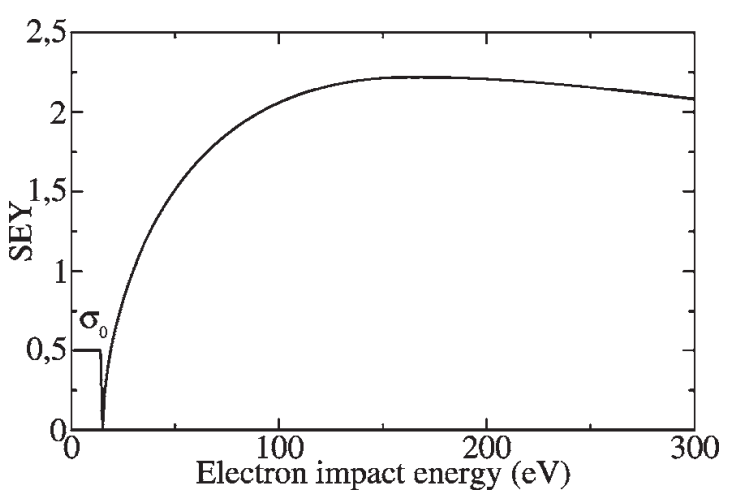

FIG. 2. SEY model for silver used in this work, extracted from Ref. 12, with nonzero SEY at low energies $\left(\sigma_{0}\right)$. In this case $\sigma_{0}=0.5$.

spect to the normal, where $W_{0}=12.5 \mathrm{eV}, k_{E}$ and $k_{\theta}$ are surface dependent parameters, and $W_{\max }$ is the energy at which the maximum SEY $\left(\sigma_{\max }\right)$ is attained.

Vaughan's formula fits the experimental data reasonably well and has been widely used for modeling multipactor. However, it does not consider the existence of low-energy elastic electrons. The presence of this kind of electrons may reduce the rate of absorption of the electron population during the OFF intervals of the multicarrier envelope, thus facilitating interperiod accumulation.

On the other hand, the Furman and Pivi SEY statistical model $^{9}$ takes into account the different nature of the electrons contributing to SEY, resulting in a SEY tending to 0.5 at low energies instead of 0 , which means that half of the low-energy electrons are reflected and the other half are absorbed. Recent SEY measurements undertaken by Cimino et $a l .{ }^{10}$ suggest that Furman and Pivi model underestimates the importance of low-energy electrons, due mainly to the lack of resolution in the experimental measurements of SEY at low energies. Cimino et al. conclude that the SEY for most materials tends to one in the limit of zero impact energy; i.e., most of the low-energy electrons are reflected.

In this work, the SEY model is a modification of the Vaughan's model ${ }^{12}$ shown in Fig. 2. According to the literature, the values of the curve at low energies $\left(\sigma_{0}\right)$ have been taken as $0,0.5$, and 1 , in order to assess its influence on the multipactor breakdown levels.

With regard to the secondary electron release velocity and angle (with respect to the normal of the surface), i.e., $v_{\mathrm{SE}}$ and $\theta_{\mathrm{SE}}$, respectively, they are assumed to be random variables with Maxwellian and cosine law distributions, respectively. ${ }^{12,20,21}$ For instance, the distribution of $v_{\mathrm{SE}}$ is

$$
f\left(v_{\mathrm{SE}}\right) \propto v_{\mathrm{SE}} \exp \left(-\frac{\left(v_{\mathrm{SE}}-v_{0}\right)^{2}}{2 v_{T}^{2}}\right),
$$

where $v_{0}$ and $v_{T}$ are the emission velocity mean and the thermal spread, respectively. On the other hand, the distribution of $\theta_{\mathrm{SE}}$ is given by

$$
f\left(\theta_{\mathrm{SE}}\right)=\sin ^{2}\left(\theta_{\mathrm{SE}}\right) \text {. }
$$

The emission angle with respect the surface tangential $\left(\phi_{\mathrm{SE}}\right)$ is uniformly distributed from 0 to $2 \pi$. 


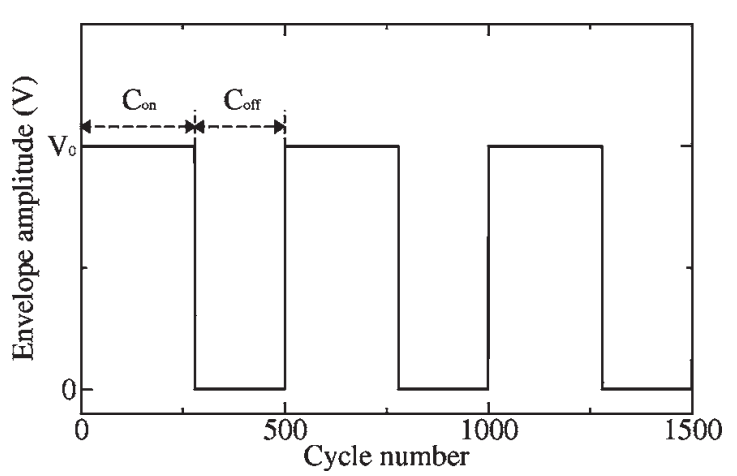

FIG. 3. Pulsed signal envelope with $C_{\text {on }}=276$ cycles and $C_{\text {off }}=224$ cycles.

\section{ACCUMULATION MODEL}

In order to investigate the charge accumulation process in the long-term multipactor discharge, it becomes necessary to study separately the electron multiplication and absorption processes during the ON and OFF times, respectively. Since the shape of the multicarrier signal envelope depends on many factors, such as the number of carriers, frequency separation and carrier phasing, it is an extremely difficult task to study the time evolution of the electron population for the general case. Therefore, let us consider a simplified envelope shape, the rectangular pulsed signal $v(t)=A(t) \sin (2 \pi f t)$, with

$$
\begin{aligned}
A(t)= & \begin{cases}V_{0}, & l\left(T_{\text {on }}+T_{\text {off }}\right)<t<(l+1) T_{\text {on }}+l T_{\text {off }}, \\
0, & (l+1) T_{\text {on }}+l T_{\text {off }}<t<(l+1)\left(T_{\text {on }}+T_{\text {off }}\right),\end{cases} \\
& l=0,1,2, \ldots,
\end{aligned}
$$

where $l$ is the envelope period number, and $V_{0}$ and $f$ are the signal amplitude and frequency, respectively. Under the pulsed signal approximation, the multicarrier signal presents a uniform ON interval during a time $T_{\text {on }}=C_{\text {on }} / 2 f$ and an OFF interval, with total absence of field, during a time $T_{\text {off }}=C_{\text {off }} / 2 f$, where $C_{\text {on }}$ and $C_{\text {off }}$ are the number of cycles for the $\mathrm{ON}$ and OFF intervals, respectively. An example of such an envelope for a particular combination of $C_{\text {on }}$ and $C_{\text {off }}$ is shown in Fig. 3.

The electrons are assumed to be in resonance during the ON interval and, therefore, it is expected that they impact against the surfaces with approximately the same energy and thus nearly the same SEY, $\sigma_{\text {on }}$. According to the classical multipactor theory, the number of electrons in the $k$ th cycle, $N(k)$, during the ON interval is

$$
N(k)=N_{i} \Gamma(k) \text { for } 0 \leq k \leq C_{\text {on }},
$$

where $N_{i}$ is the initial number of electrons (for $k=0$ ) and $\Gamma(k)$ is the electron multiplication factor, defined for the $\mathrm{ON}$ interval as

$$
\Gamma(k)=\sigma_{\text {on }}^{k / n} \quad \text { for } 0 \leq k \leq C_{\text {on }},
$$

where $k / n$ is the number of impacts for a single electron in $k$ cycles, $n$ being the two-surface multipactor order. Therefore, at the end of the ON interval the multiplication factor is

$$
\Gamma_{\mathrm{on}}=\Gamma\left(C_{\mathrm{on}}\right)=\sigma_{\mathrm{on}}^{C_{\mathrm{on}} / n} \text {. }
$$

This expression does not consider the effects of the space charge, which is mainly responsible for the saturation of the multipactor discharge. ${ }^{3}$ However, its effects manifest for high electron densities ${ }^{22}$ and, since a typical multipactor discharge initiates with a low number of free electrons, space charge can be neglected at the first stages, leaving Eq. (9) valid for the purpose of this work.

During the OFF interval, there is no applied field and, therefore, after the first impact, the surviving electrons travel from side to side of the device with a constant velocity equal to the secondary emission velocity. The component of such velocity perpendicular to the surface is given by $v_{\perp}=\cos \left(\theta_{\mathrm{SE}}\right) v_{\mathrm{SE}}$. Hence, $v_{\perp}$ is a random variable defined by the statistics of the electron emission velocity $v_{\mathrm{SE}}$ and angle $\theta_{\mathrm{SE}}$. Since the electrons are not accelerated, the impact energy is equal to the emission energy, which, for typical $v_{\mathrm{SE}}$ values (energies in the range of few eV), leads to a SEY equal to $\sigma_{0}$ (see Fig. 2). Under such circumstances, the electron population in the cycle $k+1$, i.e., $N(k+1)$, is given by

$$
N(k+1)=N(k)+i(k)\left(\sigma_{0}-1\right) .
$$

Here, $i(k)$ is the total number of impacts during the cycle $k$. From Eq. (10), the effective SEY per cycle, i.e., $\sigma_{\text {eff }}$ is defined as

$$
\sigma_{\text {eff }} \equiv\langle N(k+1) / N(k)\rangle=1+\left\langle\frac{i(k)}{N(k)}\right\rangle\left(\sigma_{0}-1\right),
$$

$\langle\cdot\rangle$ being the statistical expectation operator and $\langle i(k) / N(k)\rangle$ the average number of impacts per electron and cycle.

On the other hand, an electron leaving one of the surfaces with constant velocity $v_{\perp}$ crosses the gap $d$ in a time $d / v_{\perp}$. Therefore, the average number of cycles between two consecutive impacts $(C)$ can be expressed as follows:

$$
C=\left\langle\frac{1}{v_{\perp}}\right\rangle 2 f d \text {. }
$$

Notice that, in general, $\left\langle 1 / v_{\perp}\right\rangle \neq 1 /\left\langle v_{\perp}\right\rangle$.

Taking into account that $\langle i(k) / N(k)\rangle=1 / C$,

$$
\left\langle\frac{i(k)}{N(k)}\right\rangle=\frac{1}{2 f d\left\langle\frac{1}{v_{\perp}}\right\rangle} \text {. }
$$

Analogously to the ON interval, the number of electrons during the OFF interval is given by

$$
N(k)=N_{f} \Gamma(k) \text { for } C_{\text {on }}<k \leq C_{\text {on }}+C_{\text {off }},
$$

where $N_{f}$ is the number of electrons at the end of the ON interval $\left[N\left(C_{\text {on }}\right)\right]$. Substituting Eq. (13) in Eq. (11), the electron multiplication factor during the OFF interval can be written as

$$
\begin{aligned}
\Gamma(k)=\sigma_{\mathrm{eff}}^{k-C_{\mathrm{on}}}= & {\left[1+\frac{\sigma_{0}-1}{2 f d\left(\frac{1}{v_{\perp}}\right)}\right]^{k-C_{\mathrm{on}}} } \\
& \text { for } C_{\mathrm{on}}<k \leq C_{\mathrm{on}}+C_{\mathrm{off}} .
\end{aligned}
$$

Therefore, at the end of the OFF interval the multiplication factor is 


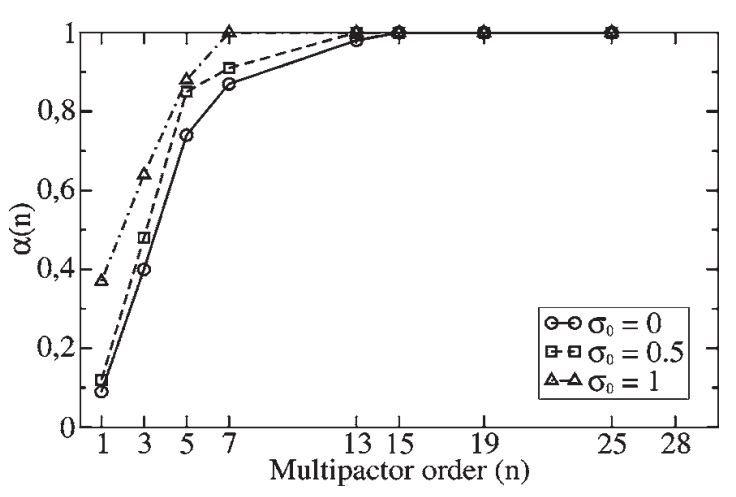

FIG. 4. Fraction $\alpha(n)$ of electrons participating in the multipactor process at the beginning of the ON interval. The curve has been numerically calculated for three different values of $\sigma_{0}$.

$$
\Gamma_{\text {off }}=\Gamma\left(C_{\text {on }}+C_{\text {off }}\right)=\left[1+\frac{\sigma_{0}-1}{2 f d\left(\frac{1}{v_{\perp}}\right\rangle}\right]^{C_{\text {off }}},
$$

which manifests the role of the SEY at low energies on the absorption rate. A $\sigma_{0}=0$ implies the maximum absorption rate (minimum $\Gamma_{\text {off }}$ ), whereas for $\sigma_{0}=1$, all impacting electrons are reflected and there is no absorption at all $\left(\Gamma_{\text {off }}=1\right)$, the electron population remaining constant.

At this point (end of the OFF interval), the surviving electrons are distributed along the waveguide with velocities following the secondary emission velocity distribution. Only the fraction of those which have the correct combination of position and velocity, in order to impact with favorable phase, will participate in the consecutive ON interval. This fraction $\alpha(n)$ varies with the multipactor mode $n$.

Figure 4 shows the values of $\alpha(n)$ for different multipactor orders and $\sigma_{0}$. The curve has been computed performing several runs of electron population versus time, calculated numerically with the multipactor simulator used in Sec. V. There are no relevant differences for the three values of $\sigma_{0}$, although higher values of it tend to raise the curve towards a higher $\alpha(n)$. The curve tends to one for increasing mode due to the fact that higher $f \times d$ products allow the simultaneous existence of different multipactor modes and hybrid modes, ${ }^{23,24}$ which, together with the spread of the secondary emission velocity and the subsequent broadening of the range of valid phases, ${ }^{13}$ enhances the probability for each electron to participate in the discharge. Indeed, for high values of $n$ (above $n=13$ ), $\alpha$ can be safely assumed to be 1 .

Finally, in order to accumulate charge, the total electron growth must hold $\left[\Gamma_{\text {on }} \Gamma_{\text {off }} \alpha(n) \geq 1\right]$, which leads, along with Eqs. (9) and (16), to the minimum average SEY value during the $\mathrm{ON}$ interval producing accumulation, i.e., $\sigma_{\mathrm{on} \text {,min }}[$ when $\left.\Gamma_{\text {on }} \Gamma_{\text {off }} \alpha(n)=1\right]$, given by

$$
\sigma_{\mathrm{on}, \min }=\left(\frac{1}{\alpha(n)}\right)^{n / C_{\mathrm{on}}}\left(1+\frac{\sigma_{0}-1}{2 f d\left(\frac{1}{v_{\perp}}\right\rangle}\right)^{-n C_{\mathrm{off}} / C_{\mathrm{on}}} .
$$

The correction factor $[1 / \alpha(n)]^{n / C_{\mathrm{on}}}$, of Eq. (17), is plotted in Fig. 5 for $\sigma_{0}=0$. Note that in most applications, typically $n / C_{\text {on }} \approx 1 / 20$ (following the 20 -gap-crossing rule),

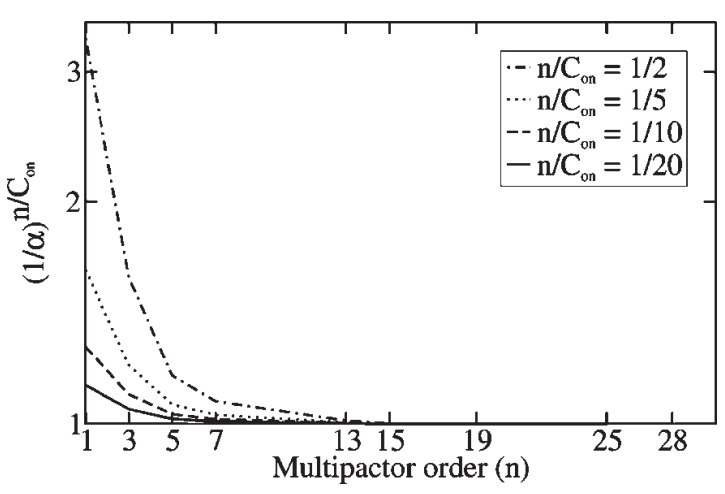

FIG. 5. Correction factor against multipactor order for fixed values of $n / C_{\text {on }}$ and $\sigma_{0}=0$.

which leads to values of this correction factor very close to 1 . Hence, it is interesting to see that, according to this model and neglecting the contribution of $\alpha(n)$, the long-term multipactor threshold $\sigma_{\text {on,min }}$, depends only on $\sigma_{0}, f \times d, n$, and $C_{\text {off }} / C_{\text {on }}=T_{\text {off }} / T_{\text {on }}$, always with $\sigma_{\text {on,min }} \geq 1$. The limit cases of $\sigma_{0}=0$ and $\sigma_{0}=1$, yield the maximum value of $\sigma_{\text {on,min }}$ and the minimum (equal to unity), respectively. This implies that the higher the value of $\sigma_{0}$, the lower the long-term multipactor threshold, which is obvious since it also implies a lower absorption rate during the OFF interval. Similarly, the $C_{\text {off }} / C_{\text {on }}$ ratio also affects the threshold in a very intuitive manner. The higher the ratio, the higher the $\sigma_{\text {on,min }}$, since the absorption process duration increases with respect to the multiplication one.

Therefore, Eq. (17) sets the long-term multipactor threshold. If a certain field envelope ensures $\sigma_{\text {on }}>\sigma_{\text {on,min }}$, there will be charge accumulation and the subsequent multipactor discharge. However, the SEY during the ON interval $\left(\sigma_{\mathrm{on}}\right)$ depends on the envelope amplitude during the ON time, since it sets the electron impact energy, and thus the SEY. This relationship cannot be easily established except for voltages close to the multipactor susceptibility threshold, it is expected that a higher ON voltage also implies a higher $\sigma_{\text {on }}$.

As stated before, the modeling of the general case for a multicarrier signal envelope is a very challenging task. However, provided that the envelope has differentiated $\mathrm{ON}$ and OFF intervals within a period with a reasonably uniform voltage for the ON time and a very low voltage for the OFF time, this pulsed signal accumulation model can be applied. For instance, the first two phase distributions of Fig. 1 meet the conditions to be modeled by a pulsed signal, whereas the arbitrary phasing of the third signal spreads its envelope throughout the whole period and thus, no differentiation between $\mathrm{ON}$ and OFF intervals can be done.

\section{NUMERICAL RESULTS}

Several numerical simulations have been carried out using the analytical solution of the electromagnetic threedimensional (3D) fields in a rectangular waveguide of infinite length for the fundamental mode $\mathrm{TE}_{10}$. An $X$-band waveguide with width $a=22.86 \mathrm{~mm}$ and height $b=2 \mathrm{~mm}$ has been chosen for the testing. The working frequency $\left(f_{m}\right.$ for 


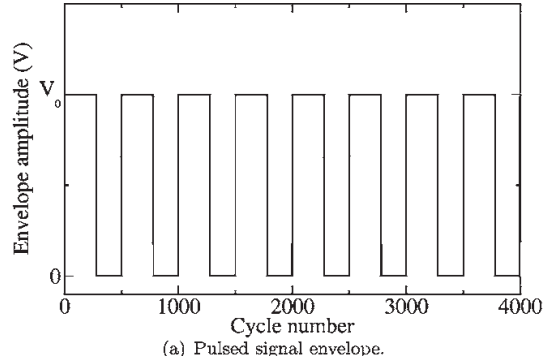

(a) PuIsed signal envelope

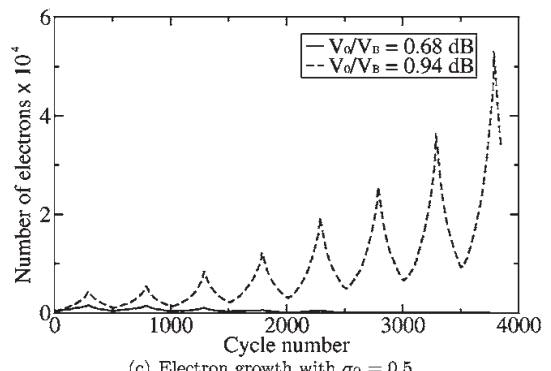

(c) Electron growth with $\sigma_{0}=0.5$

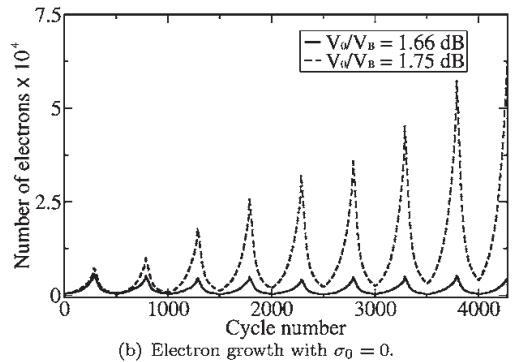

(b) Electron growth with $\sigma_{0}=0$

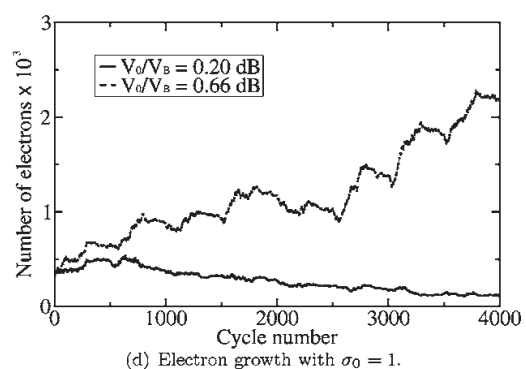

FIG 6. (a) Pulsed signal envelope, with a period $T=25 \mathrm{~ns}, T_{\text {on }}=13.8 \mathrm{~ns}$ and $T_{\text {off }}=11.2 \mathrm{~ns}$. $V_{0}$ is the ON voltage. (b) to (d) Electron population growth for different $\sigma_{0}$. The curves have been plotted for different values of $V_{0}$ to breakdown voltage ratio $V_{0} / V_{B}$, expressed in decibels. multicarrier operation) is $10 \mathrm{GHz}$, with $f \times d=20 \mathrm{GHz} \mathrm{mm}$, $n=15$, and $T_{20}=15 \mathrm{~ns}$. The 3D trajectories have been determined for each individual electron with the Velocity-Verlet algorithm, using the method of Ref. 12.

The chosen material is silver with the SEY parameters extracted from Ref. 6 where the SEY model of Ref. 12 has been applied. For a Maxwellian secondary emission energy with a thermal spread of $3 \mathrm{eV}$, and a cosine law distribution for the emission angle, the mean value $\left\langle 1 / v_{\perp}\right\rangle$ of Eq. (12) results in $2.6 \times 10^{-6} \mathrm{~s} \mathrm{~m}^{-1}$.

The numerical tests have been run with the three possible values of $\sigma_{0}$ proposed by the existing literature and mentioned in Sec. III; i.e., 0, 0.5, and 1. Two kind of input signals have been used, rectangular pulsed and multicarrier, with the parameters shown in Figs. 6 and 8, respectively.

The main reason to choose such signals is twofold. First, both signals have equivalent $\mathrm{ON}$ and $\mathrm{OFF}$ times in order to be compared. Second, the ON time should always be less than $T_{20}$ in order to ensure that the 20-gap-crossing rule is always accomplished. According to such a rule, such signals should never develop a multipactor discharge in a single peak.

\section{A. Single-carrier signal}

The multipactor breakdown voltage has been numerically computed for the single-carrier signal. Table I shows the influence of $\sigma_{0}$ on the breakdown voltage along with the fitted value from the experimental results of Ref. 6 . As it can be observed, the numerical results for $\sigma_{0}=0.5$ offer the closer value to the experimental one, which agree with the results

TABLE I. Breakdown voltage for single carrier.

\begin{tabular}{ccccc}
\hline \hline$\sigma_{0}$ & 0 & 0.5 & 1 & Experimental $^{\mathrm{a}}$ \\
\hline$V_{B}(\mathrm{~V})$ & 1635 & 1480 & 1173 & 1400 \\
\hline
\end{tabular}

$\overline{\bar{a}}$ Extracted from Ref. 6. given in Ref. 25. Notice that using a zero low-energy SEY implies an overestimation of the multipactor threshold.

\section{B. Pulsed signal}

The simulator has been run for each $\sigma_{0}$ and for a pulsed signal with $C_{\text {on }}=276, C_{\text {off }}=224$, and increasing input voltage $V_{0}$, shown in Fig. 6(a). As expected, for low values of $V_{0}$ (implies low $\sigma_{\text {on }}$ ) no accumulation is observed, but beyond a certain voltage, here defined as the accumulation voltage threshold $V_{\text {acc }}$, the time evolution of the electron population shows a noticeable interperiod accumulation. This can be seen in Figs. 6(b)-6(d), which show the electron population growth with time for two different levels of $V_{0}$ : below and equal to $V_{\text {acc }}$. Notice that the creation or absorption of electrons coincide with the $\mathrm{ON}$ and $\mathrm{OFF}$ periods of the envelope, respectively. Accumulation has been observed for the three values of $\sigma_{0}$.

The average value of $\sigma_{\text {on,min }}$ for all impacting electrons during the $\mathrm{ON}$ interval has been computed numerically for $V_{0}=V_{\text {acc }}$ and compared with the minimum value predicted by Eq. (17), assuming $\alpha(n)=1$ since the mode order of the discharge is considerably high $(n=15)$. Table II shows these results for all values of $\sigma_{0}$. A good agreement between numerical results and predictions is found. Moreover, the accumulation to breakdown margin $V_{\text {acc }} / V_{B}$ reduces as $\sigma_{0}$ increases, which implies that higher low-energy SEY values increases the susceptibility of the system to develop a multipactor accumulation process.

TABLE II. Analytical and numerical results for pulsed signal.

\begin{tabular}{lcccc}
\hline \hline & $\begin{array}{c}\sigma_{\text {on, } \min } \\
\sigma_{0}\end{array}$ & $\begin{array}{c}\sigma_{\text {on,min }} \\
\text { (numerical) }\end{array}$ & $V_{\text {acc }}(\mathrm{V})$ & $V_{\text {acc }} / V_{B}(\mathrm{~dB})$ \\
\hline 0 & 1.12 & 1.14 & 2000 & 1.75 \\
0.5 & 1.06 & 1.09 & 1650 & 0.94 \\
1 & 1.00 & 1.02 & 1265 & 0.66 \\
\hline \hline
\end{tabular}




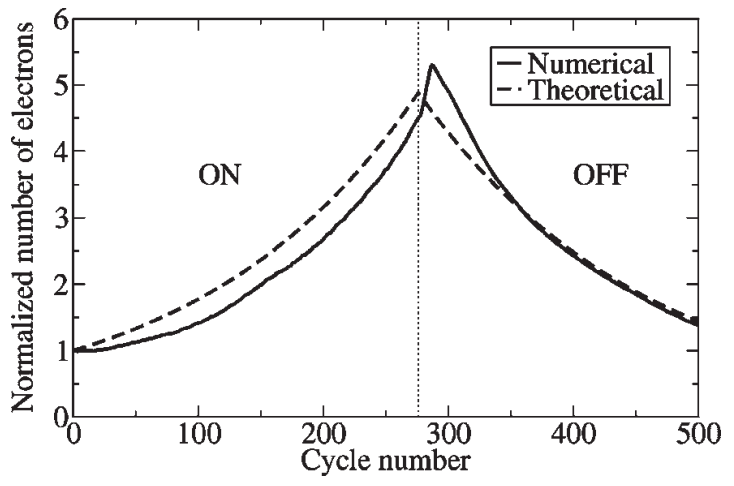

FIG. 7. Electron growth for a single period of the pulsed signal for $\sigma_{0}=0.5$. The ON and OFF intervals are delimited by the vertical dotted line.

Figure 7 shows the electron growth during a single period of the pulsed signal envelope assuming a unique initial electron and $\sigma_{0}=0.5$. Both theoretical and numerical curves are plotted. The theoretical curve is obtained using Eqs. (7), (8), (14), and (15), with the value for $\sigma_{\text {on }}$ given in Table II and $\alpha(n)=1$. The numerical simulation shows that the electrons keep on multiplicating few cycles after the end of the $\mathrm{ON}$ interval and then the absorption process starts. This is because at the end of the ON interval there is a high number of energetic electrons that are able to generate secondaries during the following cycles even if no field is present, thus maintaining the growth rate. On the other hand, the electron growth at the beginning of the ON interval in the numerical simulation is slightly delayed with respect to the theory. This is caused by the initial nonresonant electrons which prevent the population to grow at full pace until complete resonance is achieved. This delay or dwell time has been also reported in experimental multipactor tests. ${ }^{26}$ Nevertheless, although introducing some deviation, these effects do not change significantly the overall agreement between theory and simulations, being the electron creation and absorption rates during the ON and OFF intervals well fitted by the theory.
TABLE III. Analytical and numerical results for multicarrier signal.

\begin{tabular}{lcccc}
\hline \hline & $\begin{array}{c}\sigma_{\text {on, } \min } \\
{[\text { Eq. (17) }}\end{array}$ & $\begin{array}{c}\sigma_{\text {on, } \min } \\
\text { (numerical) }\end{array}$ & $V_{\text {acc }}(\mathrm{V})$ & $V_{\text {acc }} / V_{B}(\mathrm{~dB})$ \\
\hline 0 & 1.34 & - & - & - \\
0.5 & 1.16 & 1.24 & 2227 & 3.55 \\
1 & 1.00 & 1.09 & 1386 & 1.45 \\
\hline \hline
\end{tabular}

\section{Multicarrier signal}

Similarly to the pulsed signal tests, the simulator has been run for the multicarrier case and for each $\sigma_{0}$, with an input signal composed of $N=10$ carrier with triangular phasing, ${ }^{19} \Delta f=40 \mathrm{MHz}$ and increasing envelope mean lobe voltage $V_{m}$ [see Fig. 8(a)]. Figures $8(\mathrm{~b})-8(\mathrm{~d})$ show the electron population growth with time. No accumulation has been observed for $\sigma_{0}=0$ and $V_{m}$ values up to $8 \mathrm{~dB}$ higher than $V_{B}$, which are far above from the nominal $n=15$ multipactor order limits.

Table III shows the predictions and numerical results for the minimum SEY. The predictions were computed using Eq. (17) with $\alpha(n)=1$ and assuming an equivalent pulsed signal to model the multicarrier envelope, with the reasonable values of $T_{\text {on }}=\frac{1}{3} T$ and $T_{\text {off }}=\frac{2}{3} T, T=25 \mathrm{~ns}$ being the envelope period.

Since the field is not zero during the OFF interval and the electrons are accelerated out of resonance, the absorption during the OFF interval increases. This produces a slightly higher difference between theory and numerical results compared to the pulsed signal counterpart. However, good agreement among predicted and numerical results is still observed. The fact that there is no accumulation for $\sigma_{0}=0$ leads to the conclusion that, with independence of the value of the input voltage (provided that the multipactor mode order does not change), the mean SEY is not higher than the minimum established by the prediction; i.e., $\sigma_{\text {on, } \min }=1.34$.

It is important to remark that according to the 20-gap-

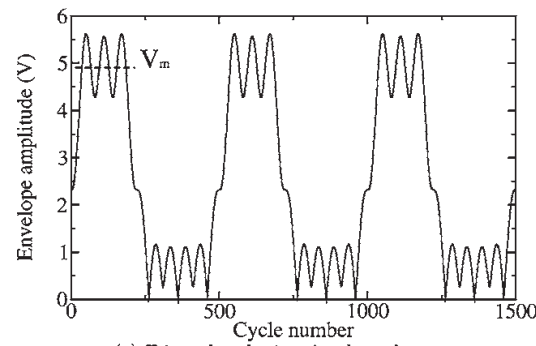

(a) Triangular phasing signal envelope.

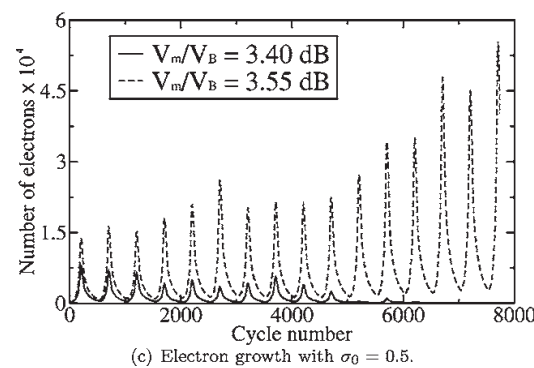

(c) Electron growth with $\sigma_{0}=0.5$

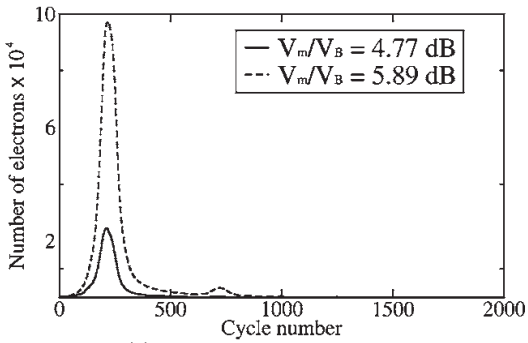

(b) Electron growth with $\sigma_{0}=0$

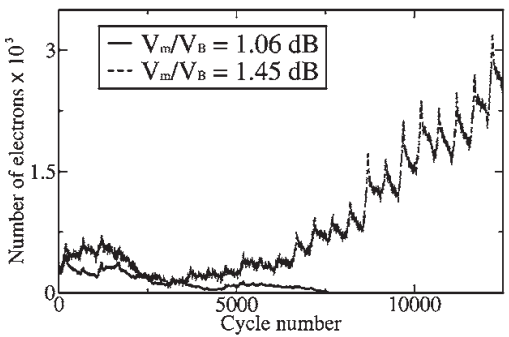

(d) Electron growth with $\sigma_{0}=1$
FIG. 8. (a) Multicarrier signal envelope $\left(V_{0}=1 \mathrm{~V}\right.$ in this example). It consists of $N=10$ carriers, with triangular phasing (Ref. 19) and $\Delta f=40 \mathrm{MHz}$, which ensures an envelope with a period of $25 \mathrm{~ns}$ and a main lobe width (from zeros) equal to $13.8 \mathrm{~ns} . V_{m}$ is the mean voltage of the envelope main lobe $\left(V_{m}=4.9 \mathrm{~V}\right.$ for $\left.V_{0}=1 \mathrm{~V}\right)$, plotted in dotted line. (b) to (d) Electron population growth for different $\sigma_{0}$. The curves have been plotted for different values of $V_{m}$ to breakdown voltage ratio $V_{m} / V_{B}$, expressed in decibels. 
crossing rule, both the pulsed and multicarrier signals should not develop a multipactor discharge in any case. However, the simulations show that beyond $V_{\text {acc }}$, the electron population increases indefinitely towards saturation due to interperiod accumulation, developing a long-term multipactor discharge.

\section{CONCLUSIONS}

By taking some simplifications on the multicarrier envelope, an analytical model of the electron population dynamics under slow varying amplitude fields has been developed. As a result, the minimum SEY during the ON period necessary to develop accumulation has been derived as a function of the multicarrier signal, material properties and multipactor order parameters.

The analytical model has been verified with a multipactor numerical simulator for a rectangular waveguide. Although the analytical model assumes an ON/OFF pulsed signal envelope shape, the numerical results demonstrate that it is also valid for more general multicarrier envelopes provided that they have a relatively uniform amplitude during the ON time, and a very low amplitude during the OFF time.

It has been demonstrated that, even if the 20-gapcrossing rule is accomplished, accumulation effect between periods of the multicarrier signal envelope may raise the electron population to significant levels producing long-term multipactor. Furthermore, a zero value of the SEY at low energies $\sigma_{0}=0$ overestimates the accumulation to breakdown margin $V_{\text {acc }} / V_{B}$, since it increases as $\sigma_{0}$ approaches zero, reaching even infinity for the multicarrier signal used.

To the knowledge of the authors, this work provides the first theoretical study for long-term multipactor in multicarrier systems, which allows us to establish a SEY threshold and predict charge accumulation for a wide variety of situations.

\section{ACKNOWLEDGMENTS}

The authors would like to thank ESA/ESTEC for having funded this research activity through the Contract "RF Breakdown in Multicarrier Systems" (19918/06/NL/GLC).
${ }^{1}$ E. W. B. Gill and A. von Engel, Proc. R. Soc. London, Ser. A 192, 446 (1948).

${ }^{2}$ A. Hatch and H. Williams, Phys. Rev. 112, 681 (1958).

${ }^{3}$ J. Vaughan, IEEE Trans. Electron Devices 35,1172 (1988).

${ }^{4}$ V. Semenov and A. Kryazhev, Phys. Plasmas 8, 5034 (2001).

${ }^{5} \mathrm{~K}$. H. Geisser and D. Wolk, in Workshop on Multipactor, RF and DC Corona and Passive Intermodulation in Space RF Hardware (ESTEC, Noordwijk, The Netherlands, 1996).

${ }^{6}$ Space Engineering: Multipacting Design and Test (ESA Publication Division, Noordwijk, The Netherlands, 2003), Vol. ECSS-20-01A

${ }^{7}$ J. Vaughan, IEEE Trans. Electron Devices 36, 1963 (1989).

${ }^{8}$ J. de Lara, F. Pérez, M. Alfonseca, L. Galán, I. Montero, E. Román, and D. Raboso, IEEE Trans. Plasma Sci. 34, 476 (2006).

${ }^{9}$ M. Furman and M. Pivi, Phys. Rev. ST Accel. Beams 5, 124404 (2002).

${ }^{10}$ R. Cimino, I. R. Collins, M. A. Furman, M. Pivi, F. Ruggiero, G. Rumolo, and F. Zimmermann, Phys. Rev. Lett. 39, 014801 (2004).

${ }^{11}$ R. Seviour, IEEE Trans. Electron Devices 52, 1927 (2005)

${ }^{12}$ C. Vicente, M. Mattes, D. Wolk, H. L. Hartnagel, J. R. Mosig, and D. Raboso, in Microwave Symposium Digest, 2005 IEEE MTT-S International, Long Beach, CA, (IEEE, New York, 2005), Vol. 2, pp.1055-1058.

${ }^{13}$ A. Sazontov, M. Buyanova, V. Semenov, E. Rakova, N. Vdovicheva, D. Anderson, M. Lisak, J. Puech, and L. Lapierre, Phys. Plasmas 12, 053102 (2005).

${ }^{14}$ R. Udiljak, D. Anderson, P. Ingvarson, U. Jordan, U. Jostell, L. Lapierre, G. Li, M. Lisak, J. Puech, and J. Sombrin, IEEE Trans. Plasma Sci. 31, 396 (2003).

${ }^{15}$ N. Rozario, H. F. Lenzing, F. Reardon, M. S. Zarro, and C. G. Baran, IEEE Trans. Microwave Theory Tech. 42,558 (1994).

${ }^{16}$ A. Sazontov, N. Vdovicheva, M. Buyanova, V. Semenov, D. Anderson, J. Puech, M. Lisak, and L. Lapierre, in Workshop on Multipactor, RF and DC Corona and Passive Intermodulation in Space RF Hardware (ESTEC, Noordwijk, The Netherlands, 2003).

${ }^{17} \mathrm{~S}$. Riyopoulos, D. Chernin, and D. Dialetis, Phys. Plasmas 2, 3194 (1995).

${ }^{18}$ L. Friederichs, in Workshop on Multipactor, RF and DC Corona and Passive Intermodulation in Space RF Hardware (ESTEC, Noordwijk, The Netherlands, 2000), pp. 79-84.

${ }^{19}$ D. Wolk, D. Schmitt, and T. Schlipf, in Workshop on Multipactor, RF and DC Corona and Passive Intermodulation in Space RF Hardware (ESTEC, Noordwijk, The Netherlands, 2000), pp. 85-91.

${ }^{20}$ N. K. Vdovicheva, A. G. Sazontov, and V. E. Semenov, Radiophys. Quantum Electron. 47, 580 (2004).

${ }^{21}$ J. Greenwood, Vacuum 67, 217 (2002).

${ }^{22}$ S. Riyopoulos, Phys. Plasmas 4, 1448 (1997).

${ }^{23}$ A. Kryazhev, M. Buyanova, V. Semenov, D. Anderson, M. Lisak, J. Puech, L. Lapierre, and J. Sombrin, Phys. Plasmas 11, 4736 (2002).

${ }^{24}$ A. Gilardini, J. Appl. Phys. 9, 4629 (1992).

${ }^{25}$ C. Vicente, M. Mattes, D. Wolk, H. L. Hartnagel, J. R. Mosig, and D. Raboso, in Workshop on Multipactor, $R F$ and DC Corona and Passive Intermodulation in Space RF Hardware (ESTEC, Noordwijk, The Netherlands, 2005, pp. 11-17.

${ }^{26} \mathrm{D}$. Raboso and A. Woode, in 25th European Microwave Conference, Bologna, Italy, (IEEE, New York, 1995), Vol. 1, pp. 190-193. 